\title{
TDPAC studies of interaction between He and A = 140 elements in $\mathrm{Fe}$
}

AUTHOR(S):

Ohkubo, Y.; Taniguchi, A.; Xu, Q.; Tanigaki, M.; Sato, $\mathrm{K}$.

\section{CITATION:}

Ohkubo, Y.... [et al]. TDPAC studies of interaction between He and A = 140 elements in Fe. Hyperfine Interactions 2014, 230(1): 187-193

\section{ISSUE DATE:}

2014-11-11

URL:

http://hdl.handle.net/2433/201957

\section{RIGHT:}

The final publication is available at Springer via http://dx.doi.org/10.1007/s10751-014-1085-9.; The full-text file will be made open to the public on 11 November 2015 in accordance with publisher's 'Terms and Conditions for Self-

Archiving':; This is not the published version. Please cite only the published version.; この論文は出版社版でありません 。引用の際には出版社版をご確認ご利用ください。 


\title{
TDPAC studies of interaction between He and $A=140$ elements in Fe
}

\section{Y. Ohkubo · A. Taniguchi $\cdot$ Q. Xu • M. Tanigaki $\cdot$ K. Sato}

\begin{abstract}
Room-temperature time-differential perturbed-angular-correlation (TDPAC) spectra of ${ }^{140} \mathrm{Ce}$ in an Fe foil and in a He-doped Fe foil, unannealed and annealed in vacuum at various temperatures, were taken in order to see whether $\mathrm{Ce}$ and $\mathrm{He}$ form in Fe a complex that has a definite geometrical structure, as suggested in first-principles density functional theory calculations. Our TDPAC results do not indicate the existence of such a complex in Fe. We also discuss briefly He-vacancy clusters formed in the bombardments of He and ${ }^{140} \mathrm{Cs}$ (a precursor of $\left.{ }^{140} \mathrm{Ce}\right)$.
\end{abstract}

Keywords $\mathrm{He} \cdot{ }^{140} \mathrm{Ce} \cdot$ ion implantation $\cdot$ defects $\cdot \mathrm{Fe} \cdot \mathrm{TDPAC}$

Y. Ohkubo $(\bowtie) \cdot$ A. Taniguchi $\cdot$ Q. Xu $\cdot$ M. Tanigaki $\cdot$ K. Sato

Research Reactor Institute, Kyoto University, Kumatori, Sennan, Osaka 590-0494, Japan

e-mail: ohkubo@rri.kyoto-u.ac.jp

\section{Introduction}

Understanding the behavior of $\mathrm{He}$ atoms in metals, especially in $\mathrm{Fe}$, is one of important research subjects in the field of nuclear fusion technology. It has been predicted from firstprinciples density-functional theory calculations that in bcc Fe, Ce has a stronger attraction to He $(-1.31 \mathrm{eV} /$ atom pair) than He does to another He $(-1.18 \mathrm{eV} / \mathrm{atom}$ pair $)$ and as a result a local structure is formed by a tetrahedron Ce cage encapsulating a He atom, suggesting that Ce, La having an electronic structure similar to $\mathrm{Ce}$, and possibly some other rare-earth elements could 
pin down mobile He atoms and thereby impede the He bubble growth supposedly produced in the steel of a fusion reactor [1]. Recently, utilizing the online isotope separator at the Research Reactor Institute, Kyoto University (KUR-ISOL) [2], we implanted ${ }^{140} \mathrm{Cs}$ in an Fe foil at room temperature and successfully observed an oscillation pattern due to a unique magnetic hyperfine interaction at ${ }^{140} \mathrm{Ce}$ arising from those ${ }^{140} \mathrm{Cs}$ via ${ }^{140} \mathrm{Ba}$ and then ${ }^{140} \mathrm{La}$ in its room-temperature time-differential perturbed-angular-correlation (TDPAC) spectrum [3]. (From the known magnetic hyperfine field at ${ }^{141} \mathrm{Ce}$ in $\mathrm{Fe}$, we have obtained the magnetic moment, $\mu$, of the 2083$\mathrm{keV}$ state of ${ }^{140} \mathrm{Ce}$.) Applying this result, in order to examine whether Ce (or rather, La and Ba, which are parent atoms of ${ }^{140} \mathrm{Ce}$ ) and He form complexes having a definite geometrical structure in Fe, as suggested by the first-principles density-functional theory calculations, we took roomtemperature TDPAC spectra of ${ }^{140} \mathrm{Ce}$ in a He-doped Fe foil (He ions were implanted after ${ }^{140} \mathrm{Cs}$ ion implantation), unannealed and annealed in vacuum at various temperatures. The key results we obtained are 1) the parent atoms ( $\mathrm{La}$ and $\mathrm{Ba}$ ) of ${ }^{140} \mathrm{Ce}$ very efficiently trap He and 2) they form complexes in Fe, but the complexes do not have a definite geometrical structure [4].

In this study, to still search for ${ }^{140} \mathrm{Ce}-\mathrm{He}$ complexes having a definite geometrical structure in Fe, we prepared two Fe samples, one by implanting only $100-\mathrm{keV}{ }^{140} \mathrm{Cs}$ and the other by implanting 4-keV He and then $100-\mathrm{keV}^{140} \mathrm{Cs}$, this doping order being opposite to the case in [4], and took room-temperature TDPAC spectra of ${ }^{140} \mathrm{Ce}$ in both the Fe foil and the He-doped Fe foil (He ions were implanted before ${ }^{140} \mathrm{Cs}$ ion implantation), each unannealed and annealed in vacuum at various temperatures. We compare the two sets of TDPAC spectra with each other, also compare the TDPAC results for the present He-doped Fe foil with relevant results of a thermal helium desorption spectroscopy (THDS) experiment [5] and mention the stability of the He-vacancy clusters produced in Fe referring to molecular dynamics calculations [6, 7].

\section{Experimental procedures}

Two Fe samples were prepared: one is Fe irradiated with $100-\mathrm{keV}^{140} \mathrm{Cs}^{+}$(about $0.7 \times 10^{12}$ ions $/ 0.2 \mathrm{~cm}^{2}$ ) and the other is Fe irradiated with $4-\mathrm{keV} \mathrm{He}^{+}$(about $2 \times 10^{16} \mathrm{ions} / \mathrm{cm}^{2}$ ) and subsequently with $100-\mathrm{keV}^{140} \mathrm{Cs}^{+}$(about $0.7 \times 10^{12}$ ions $/ 0.2 \mathrm{~cm}^{2}$ ). This dose of He corresponds to $0.15 \mathrm{dpa}$ (displacement per atom) at the maximum. We name the first sample ${ }^{140} \mathrm{CeFe}$ and the second ${ }^{140} \mathrm{Ce}$ He-doped Fe. (Note that ion implantation is the only way to incorporate La or Ce in 
Fe because they do not form a solid solution with Fe.) Each Fe foil was of size $10 \mathrm{~mm} \times 10 \mathrm{~mm} \times$ $0.1 \mathrm{~mm}$ and of $99.995 \%$ purity, annealed in $\mathrm{H}_{2}$ atmosphere at $973 \mathrm{~K}$ for $2 \mathrm{~h}$ and then electropolished. Room-temperature projections of ${ }^{140} \mathrm{Cs}$ ions were performed at the solid-state physics beam course of KUR-ISOL [2]. These ${ }^{140} \mathrm{Cs}$ were obtained by the fission reaction of a 50$\mathrm{mg}{ }^{235} \mathrm{U}$ target with thermal neutrons of $3 \times 10^{12} / \mathrm{cm}^{2} \mathrm{~s}$ and were carried to the surface ionizer by $\mathrm{PbI}_{2}$ aerosol in a mixed gas jet of $\mathrm{He}$ and $\mathrm{N}_{2}$. The ionized fission products were accelerated to 30 $\mathrm{keV}$ for mass analysis. Mass-separated ${ }^{140} \mathrm{Cs}$ ions were further accelerated to $100 \mathrm{keV}$ by the post accelerator, successively decaying through ${ }^{140} \mathrm{Ba}$ and then ${ }^{140} \mathrm{La}$ into ${ }^{140} \mathrm{Ce}$ as shown in Fig. 1 [8]. The He implantation was carried out at room temperature in vacuum of $8 \times 10^{-6} \mathrm{~Pa}$ using an ion beam gun manufactured by Omegatron Co. in which mono-energetic 4-keV $\mathrm{He}^{+}$ions were collimated and mass-analyzed [9]. Figure 2 shows that the distribution profile of $\mathrm{He}$ in Fe overlaps that of ${ }^{140} \mathrm{Cs}$, both of which were calculated with a SRIM code [10].

For the ${ }^{140} \mathrm{CeFe}$ and ${ }^{140} \mathrm{CeHe}$-doped Fe samples, 0.5-h annealing in vacuum (about $1 \times 10^{-4}$ $\mathrm{Pa}$ ) was performed at a same time in a same furnace at 473, 673, 773, 873, 973 and $1073 \mathrm{~K}$ in this order. Before and after each annealing, the time dependences of the coincidence counts $N(\theta$, t) of the 329-487 keV cascade $\gamma$ rays for the two Fe samples were taken at room temperature with two measurement systems, each consisting of standard fast-slow electronic modules and four $\mathrm{BaF}_{2}$ scintillation detectors. Here, $\theta$ and $t$ denote the angle and the time interval, respectively, between the cascade $\gamma$ rays. The directional anisotropy $A_{22} G_{22}(t)$ is obtained as follows:

$$
A_{22} G_{22}(t)=2 \frac{N\left(180^{\circ}, t\right)-N\left(90^{\circ}, t\right)}{N\left(180^{\circ}, t\right)+2 N\left(90^{\circ}, t\right)} .
$$

The $10 \mathrm{~mm} \times 10 \mathrm{~mm}$ irradiation plane of each Fe foil was set parallel to the detector plane. The distance between the center of the source and the head of each $\mathrm{BaF}_{2}$ detector was $3.0 \mathrm{~cm}$. With four detectors, slight differences in the $\gamma$-ray detection efficiency among the detectors are eliminated in the operation of (1) [3].

The coefficient $A_{22}$ depends only on the nuclear transitions and its value for ${ }^{140} \mathrm{Ce}$ is reported to be -0.13 [11]. The perturbation factor $G_{22}(t)$ for an ensemble of randomly oriented microcrystals is a function of the Larmor frequency $\omega_{\mathrm{L}}$ for a unique static magnetic interaction. The $\omega_{\mathrm{L}}$ is defined as usual: $\omega_{\mathrm{L}}=-\mu B / I \hbar$, where $B$ is the magnetic field at the nucleus. In the $180^{\circ}-90^{\circ}$ angular correlation case with no external magnetic field, 


$$
A_{22} G_{22}(t)=A_{22}\left[1+2 \cos \left(\omega_{\mathrm{L}} t\right)+2 \cos \left(2 \omega_{\mathrm{L}} t\right)\right] / 5,
$$

having the constant term (which we call the baseline in the text) of $A_{22} / 5$.

All the TDPAC measurements were done more than about a week after the end of either ${ }^{140} \mathrm{Cs}$ bombardment so that the number ratio of ${ }^{140} \mathrm{La}$ to ${ }^{140} \mathrm{Ba}$ always equals $t_{1 / 2}\left({ }^{140} \mathrm{La}\right) /\left[t_{1 / 2}\left({ }^{140} \mathrm{Ba}\right)-\right.$ $\left.t_{1 / 2}\left({ }^{140} \mathrm{La}\right)\right]=0.15$ during the TDPAC measurements, where $t_{1 / 2}\left({ }^{140} \mathrm{La}\right)=40.3 \mathrm{~h}$ and $t_{1 / 2}\left({ }^{140} \mathrm{Ba}\right)=$ $12.8 \mathrm{~d}$.

\section{Results and discussion}

Figure 3 displays most of the $A_{22} G_{22}(t)$ time spectra obtained at room temperature for the two samples, (a) ${ }^{140} \mathrm{CeFe}$ and (b) ${ }^{140} \mathrm{CeHe}$-doped Fe. For each sample, the top panel represents the spectrum before annealing, and the second, the third, the fourth and the bottom panels represent those after annealing in vacuum at 473, 673, 773 and $1073 \mathrm{~K}$, respectively. As explained in [3], the oscillation pattern seen in the top panel of Fig. 3(a) is due to a unique static magnetic hyperfine interaction at ${ }^{140} \mathrm{Ce}$ occupying the Fe substitutional site with no lattice defects nearby (the Larmor frequency $\omega_{\mathrm{L}}$ equals about $1.9 \mathrm{Grad} / \mathrm{s}$ ). ${ }^{1}$ Note that the oscillation amplitude does not get damped with time and this feature is common to the other panels of Fig. 3 that show the oscillation pattern. ${ }^{2}$ These ${ }^{140} \mathrm{Ce}$ experience no electric quadrupole interaction and are diamagnetic (Ce ${ }^{4+}$ with no $4 f$ electron). Henceforth, we call them "good" ${ }^{140} \mathrm{Ce}$.

${ }^{1}$ The top spectrum of Fig. 3(a) in the text is not the one displayed in [3]. But, these two spectra are identical to each other with respect to the oscillation frequency: for the former $\omega_{\mathrm{L}}=1.930(8)$ Grad/s and for the latter in [3] $\omega_{\mathrm{L}}=1.927(7) \mathrm{Grad} / \mathrm{s}$, although the amplitude seems dependent on the batch of Fe foils: -0.053(3) for the former and -0.042(3) for the latter.

${ }^{2}$ Although according to (2), there should be two frequency components for one magnetic interaction, there appears to be only one frequency component for the oscillation pattern. This is due to the finite time resolution of the present detection systems, 1 ns, about 10 times larger than the time bin of the time spectra. The third term of (2) being proportional to $\cos \left(2 \omega_{\mathrm{L}} t\right)$ is almost averaged out. 
In addition to this oscillation component, other components contribute to the TDPAC spectrum (top panel in Fig. 3(a)). Because the amplitude of the oscillation is -0.053 , while the reported $A_{22}$ value is -0.13 , the oscillation component corresponds to about $40 \%$ of the ${ }^{140} \mathrm{Ce}$ implanted in $\mathrm{Fe}$ and therefore the other components about 60\%. From the observation that the baseline of the TDPAC spectrum is nearly equal to $A_{22} / 5(=-0.026)$, which is the first term of (2), we see that these components are due to static perturbations. ${ }^{3}$ Considering that there are events in which atomic vacancies produced during the $100-\mathrm{keV}{ }^{140} \mathrm{Cs}$ bombardment migrate and bond to ${ }^{140} \mathrm{Cs}\left(t_{1 / 2}=63.7 \mathrm{~s}\right)$ and possibly ${ }^{140} \mathrm{Ba}$, these components arise from those ${ }^{140} \mathrm{Ce}$ at the Fe sites having lattice defects nearby, or at interstitial sites or in grain boundaries, feeling a variety of static hyperfine magnetic fields and electric field gradients such that the oscillations arising from these various fields interfere among themselves and get damped quickly in the TDPAC spectrum.

In Fig. 3 are shown the values of the $\omega_{\mathrm{L}}$ (in Grad/s) and the amplitude for each oscillation component in the TDPAC spectra, which were obtained by fitting (2) taking account of the time resolution of the measurement systems. The TDPAC spectra for the unannealed ${ }^{140} \mathrm{CeFe}$ (top panel in Fig. 3(a)) and ${ }^{140} \mathrm{CeHe-doped} \mathrm{Fe} \mathrm{(top} \mathrm{panel} \mathrm{in} \mathrm{Fig.} \mathrm{3(b))} \mathrm{are} \mathrm{identical} \mathrm{to} \mathrm{each} \mathrm{other:} \mathrm{no}$ He doping effect is seen in this TDPAC spectrum for ${ }^{140} \mathrm{CeHe}$-doped Fe. Now, we look at Fig. 3(a) downwards. The oscillation amplitude for ${ }^{140} \mathrm{CeFe}$ gets reduced by about $20 \%$ at the annealing temperature of $473 \mathrm{~K}$ and by about $50 \%$ at $673 \mathrm{~K}$, at which temperature the pattern of the other components described above exhibits a simply decreasing time dependence in the time region below about $10 \mathrm{~ns}$. At the annealing temperature of $773 \mathrm{~K}$, there seems to be no oscillation component in the spectrum for ${ }^{140} \mathrm{CeFe}$, i.e., there is no "good" ${ }^{140} \mathrm{Ce}$ in ${ }^{140} \mathrm{CeFe}$. There are only the other components; their $A_{22} G_{22}$ decreases monotonically with time to a constant value close to the baseline of $A_{22} / 5(=-0.026)$. This time dependence diminishes at $1073 \mathrm{~K} ; A_{22} G_{22}$ does not reach the baseline in the time range observed. Then, we look at Fig. 3(b). Very different from the case of ${ }^{140} \mathrm{CeFe}$, in the case of ${ }^{140} \mathrm{CeHe}$-doped Fe, the oscillation amplitude is not reduced at all until at $673 \mathrm{~K}$ at least: all "good" ${ }^{140} \mathrm{Ce}$ remain "good". At the annealing temperature of $773 \mathrm{~K}$ the oscillation amplitude gets reduced by about $60 \%$. At $1073 \mathrm{~K}$, the oscillation component seems to disappear in the spectrum (the other components described above exhibit a somewhat more

\footnotetext{
${ }^{3}$ In the case of a dynamic perturbation, $G_{22}(t)$ exponentially decreases to zero with $t$.
} 
rapidly decreasing time dependence in the time region below about 5 ns than those for ${ }^{140} \mathrm{CeFe}$ annealed at $1073 \mathrm{~K})$.

The observation that all "good" ${ }^{140} \mathrm{Ce}$ remain "good" in ${ }^{140} \mathrm{CeHe}$-doped Fe until at $673 \mathrm{~K}$ and about $60 \%$ of them do not at $773 \mathrm{~K}$ means that in ${ }^{140} \mathrm{CeHe}$-doped Fe, parent atoms of "good" ${ }^{140} \mathrm{Ce}$ do not migrate and any atomic defect does not migrate and bond to parent atoms of "good" ${ }^{140}$ Ce below a temperature between 673 and $773 \mathrm{~K}$ (we call it $T_{\mathrm{b}}, 673 \mathrm{~K}<T_{\mathrm{b}}<773 \mathrm{~K}$ ). This, on the other hand, indicates the cause of the oscillation amplitude diminishing at 473 and $673 \mathrm{~K}$ in the TDPAC spectra for ${ }^{140} \mathrm{CeFe}$ : because parent atoms of "good" ${ }^{140} \mathrm{Ce}$ do not migrate at these temperatures, atomic vacancies (mainly produced in the ${ }^{140} \mathrm{Cs}$ bombardment) must migrate and bond to parent atoms of "good" ${ }^{140} \mathrm{Ce}$, decreasing the number of "good" ${ }^{140} \mathrm{Ce}$. Then, we reach the conclusion that in ${ }^{140} \mathrm{CeHe}$-doped Fe, predoped He bond to vacancies mainly produced in both the He and ${ }^{140} \mathrm{Cs}$ bombardments, forming He-vacancy clusters so that neither He nor vacancies can migrate at temperatures below $T_{\mathrm{b}}$.

Let us compare our TDPAC results for ${ }^{140} \mathrm{CeHe}$-doped Fe with relevant THDS results obtained by Morishita et al. [5]. They irradiated Fe foils at room temperature with 8-keV $\mathrm{He}^{+}$ ions with the dose ranging from $2 \times 10^{13}$ to $2 \times 10^{16} / \mathrm{cm}^{2}$ and then detected He released from the foils while heated up to $1500 \mathrm{~K}$ with the fixed ramping rates of the temperature between 1 and 10 $\mathrm{K} / \mathrm{s}$. The thermal desorption spectra show a sharp peak at $450 \mathrm{~K}$ (peak I), two broad peaks at 700 800 K (peak II) and 800 1000 K (peak IV), a sharp peak at $1100 \mathrm{~K}$ (peak III) and peaks at temperatures higher than $1250 \mathrm{~K}$ (peak V) (At the dose of the order of $10^{13} / \mathrm{cm}^{2}$ were observed only the peaks I, II and III. All the He atoms were released by heating up to about $1500 \mathrm{~K}$.) Here, we are concerned with the peaks I, II and IV. The peak I at $450 \mathrm{~K}$ was assigned to He released from vacancies near the surface. Because there is essentially no difference between the TDPAC spectrum for the unannealed ${ }^{140} \mathrm{CeHe}$-doped Fe and that for the $473-\mathrm{K}$ annealed one, we consider that these He and vacancies near the surface rarely migrate to parent atoms of ${ }^{140} \mathrm{Ce}$, which seems to be consistent with the number ratio of He to ${ }^{140} \mathrm{Cs}$ near the surface being much larger than that at the peak depth around $20 \mathrm{~nm}$, as seen in Fig. 2. The peak II at 700 800 K was assigned to He released from He-vacancy clusters $\left(\mathrm{He}_{n} \mathrm{~V}\right.$, where $2 \leq n \leq 6$ and $\mathrm{V}$ stands for vacancy). The dissociation energy is about $2.2 \mathrm{eV}[5,6]$. Since $T_{\mathrm{b}}$ is in $700 \sim 800 \mathrm{~K}$, these He may migrate and bond to parent atoms of ${ }^{140} \mathrm{Ce}$, diminishing “good” ${ }^{140} \mathrm{Ce}$ by forming clusters that take a variety of 
geometrical structures. The peak IV at $800 \sim 1000 \mathrm{~K}$ was assigned to He released from Hevacancy clusters $\left(\mathrm{He}_{n} \mathrm{~V}_{m}\right)$, which we cannot identify in the TDPAC spectra.

We think that He-vacancy clusters in which He are more abundant than vacancies are formed during the He (about $2 \times 10^{16} / \mathrm{cm}^{2}$ ) and ${ }^{140} \mathrm{Cs}$ (about $0.7 \times 10^{12} / 0.2 \mathrm{~cm}^{2}$ ) bombardments and also during the heat treatments and that because the vacancy dissociation energy is larger than the He dissociation energy for such He-vacancy clusters, as shown by molecular dynamics calculations $[6,7]$, at annealing temperatures higher than $T_{\mathrm{b}}$, He are more easily released from these Hevacancy clusters than vacancies, migrating and bonding to parent atoms of ${ }^{140} \mathrm{Ce}$. At the $n / m$ value smaller than some value $(\approx 1.8$ in [6] and $\approx 1.3$ in [7]), then vacancies may get more easily released than He.

Finally, we state that also in the present doping process (first He and second ${ }^{140} \mathrm{Cs}$ ), as in the previous doping process in [4], we did not observe ${ }^{140} \mathrm{Ce}-\mathrm{He}$ complexes having any definite geometrical structure in Fe. If ${ }^{140} \mathrm{Ce}-\mathrm{He}$ complexes exist that take one or a few definite geometrical structures, these would be reflected in the TDPAC spectra for ${ }^{140} \mathrm{CeHe}$-doped Fe. But, the only clear signal is the undamped oscillation with $\omega_{\mathrm{L}}$ being equal to about $1.9 \mathrm{Grad} / \mathrm{s}$ for "good" ${ }^{140} \mathrm{Ce}$.

\section{Acknowledgments}

The present work was accomplished as part of the Project Research (25P2-4) of the Kyoto University Research Reactor Institute (KURRI). 


\section{References}

1. Hao W., Geng, W.T.: Nucl. Instrum. Methods Phys. Res. B 280, 22 (2012)

2. Okano, K., Kawase, Y., Kawade, K., Yamamoto, H., Hanada, M., Katoh, T., Fujiwara, I.: Nucl. Instrum. Methods Phys. Res. 186, 115 (1981); Kawase, Y., Okano, K., Funakoshi, F.: Nucl. Instrum. Methods Phys. Res. A 241, 305 (1985)

3. Ohkubo, Y., Taniguchi, A., Xu, Q., Tanigaki, M., Shimizu, N., Otsuka, T.: Phys. Rev. C 87, 044324 (2013)

4. Ohkubo, Y., Taniguchi, A., Xu, Q., Tanigaki, M., Sato, K.: Phil. Mag. Lett. 94, 470 (2014)

5. Morishita, K., Sugano, R., Iwakiri, H., Yoshida, N., Kimura, A.: Proc. 4th Pacific Rim Int. Conf. on Advanced Materials and Processing (PRICM4), The Japan Institute of Metals (2001), pp. 1395-1398

6. Morishita, K., Sugano, R., Wirth, B.D., Diaz de la Rubia, T.: Nucl. Instrum. Methods Phys. Res. B 202, 76 (2003)

7. Fu, C.-C., Willaime, F.: Phys. Rev. B. 72, 064117 (2005)

8. Firestone, R.B.: Table of Isotopes, 8th ed., Shirley, V. S. ed., Wiley, New York, 1996, Vol. 1

9. Xu, Q., Yamasaki, H., Sato, K., Yoshiie, T.: Phil. Mag. Lett. 91, 724 (2011)

10. Ziegler, J.F., Ziegler, M.D., Biersak, J.P.: Nucl. Instrum. Methods Phys. Res. B 268, 1818 (2010). Available at http://www.SRIM.org

11. Klemme, B., Miemczyk, H.: J. Phys. Soc. Jpn. 34 Suppl., 265 (1973) 


\section{Figure Captions}

Fig. 1. Simplified decay scheme of the relevant $A=140$ mass chain. The $2083-\mathrm{keV}$ state of ${ }^{140} \mathrm{Ce}$ used for the TDPAC measurements is shown which has a nuclear spin and parity $I^{\pi}=4^{+}$and a half-life $t_{1 / 2}=3.4 \mathrm{~ns}$, together with the $329-487 \mathrm{keV}$ cascade $\gamma$ rays

Fig. 2. Distributions of $100-\mathrm{keV}{ }^{140} \mathrm{Cs}(\bullet)$ and $4-\mathrm{keV}$ He (०) in Fe. Note that in the actual irradiations, the number of ${ }^{140} \mathrm{Cs}$ is much smaller than that for He

Fig. 3. Room-temperature TDPAC spectra for (a) ${ }^{140} \mathrm{CeFe}$ and (b) ${ }^{140} \mathrm{CeHe}$-doped Fe, subjected to no annealing, 0.5-h annealing in vacuum at 473, 673, 773 and $1073 \mathrm{~K}$ (after annealed at 873 and $973 \mathrm{~K})$. The two values in the square brackets in each panel are the Larmor frequency in units of Grad/s and the amplitude of the oscillation component, obtained by fitting (2) in the text to the spectrum taking into account the finite time resolution of the measurement systems. The number in each parentheses in either top panel is the uncertainty attached to the last digit of the value of the relevant quantity. The same uncertainties are applied to the other cases 


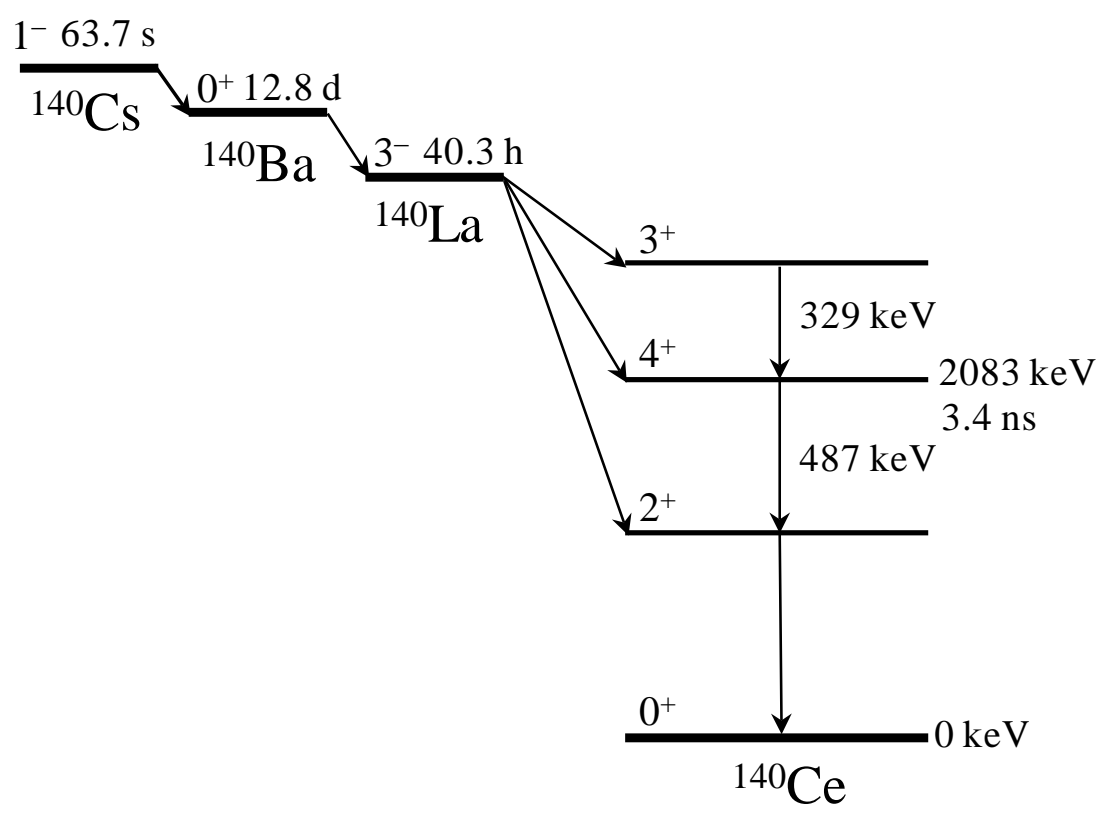

Figure 1 Ohkubo et al. 


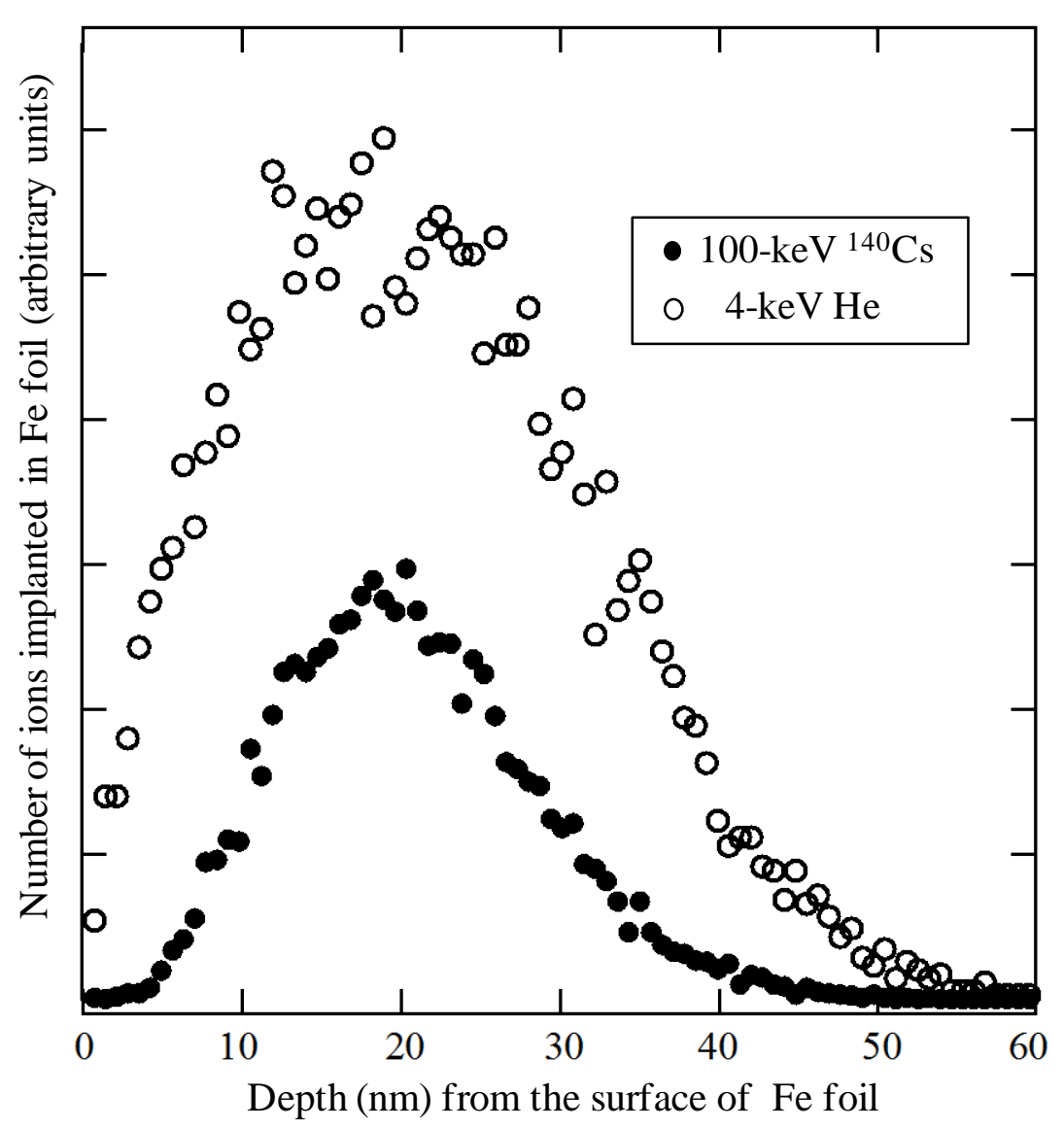

Figure 2 Ohkubo et al. 
(a) ${ }^{140} \mathrm{CeFe}$

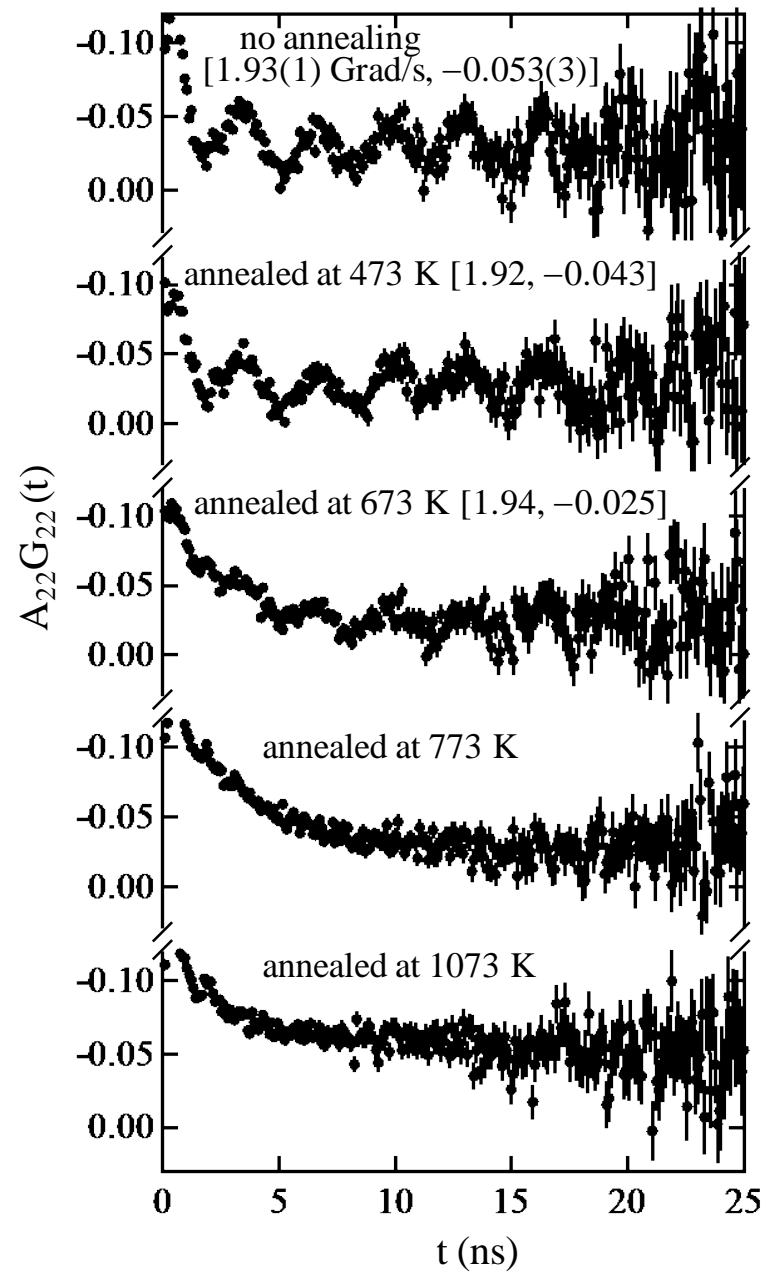

(b) ${ }^{140} \mathrm{CeHe}$-doped Fe

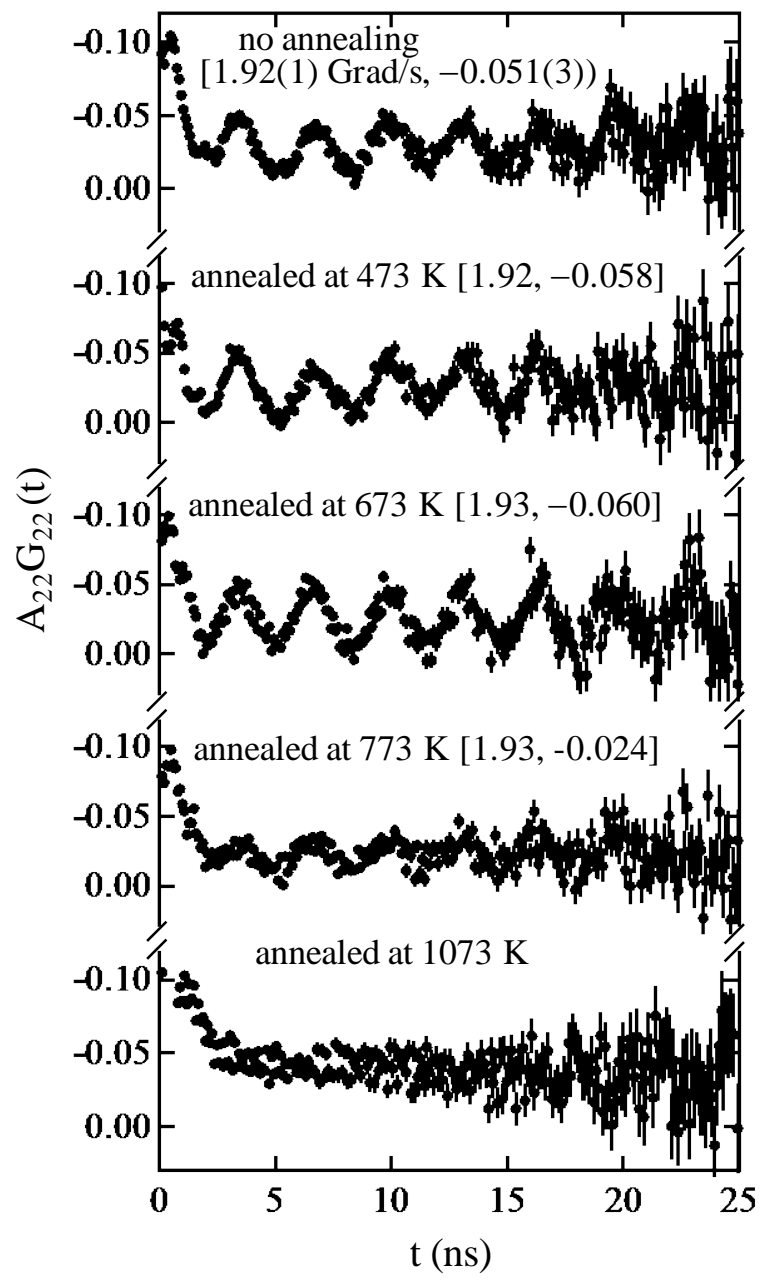

Figure 3 Ohkubo et al. 